\title{
Segmentation of Acute Lymphoblastic Leukemia Using C-Y Color Space
}

\author{
Reham Mohammed \\ Dept of Computer Science, \\ Faculty of Computers and \\ Information \\ Mansoura University, Egypt
}

\author{
Omima Nomir \\ Dept of Computer Science, \\ Faculty of Computers and \\ Information \\ Mansoura University, Egypt
}

\author{
Iraky Khalifa \\ Dept of Computer Science, \\ Faculty of Computers and \\ Information, \\ Helwan University, Egypt
}

\begin{abstract}
Medical image analysis process usually starts with segmentation step, which aims to separate different objects in the image scene. This is achieved by mainly dividing the image into two parts, the region of interest (ROI) and the background. Segmentation of acute lymphoblastic leukemia blood cell (ALL) based on microscope color image is one of the important step in the recognition process. This paper proposed a technique which aims to segment the color image of acute leukemia by transforming the RGB color space to $\mathrm{C}$-Y color space .in the $\mathrm{C}$ $\mathrm{Y}$ color space, the luminance component is used to segment (ALL) .The proposed algorithm runs on 100 microscopic ALL images and the experimental result shows that the proposed system can provide a good segmentation of ALL from its complicated background and shows that the segmentation accuracy of the proposed technique is $98.38 \%$ compared to the result of the manual segmentation method by expert.
\end{abstract}

Keywords-Image Segmentation; acute lymphoblastic leukemia; RGB; C-Y color space

\section{INTRODUCTION}

Cancer is a class of diseases characterized by out-ofcontrol cell growth. There are many different types of cancer, and each is classified by the type of cell that is initially affected, one of them is Leukemia. Leukemia is a cancer that begins in the bone marrow. It is caused by excessive production of leucocytes that replace normal blood cells. There are four major different types of Leukemia according to the growth speed overproduction of leukemic cells [1]. The four main types of Leukemia are : Acute lymphoblastic leukemia (ALL) Acute myelogenous leukemia(AML),Chronic lymphocytic leukemia(CLL) and Chronic myelogenous leukemia (CML).This work focuses on the segmentation of Acute lymphoblastic leukemia (ALL), which is called also childhood leukemia.The early and fast identification of the leukemia type, greatly aids in providing the appropriate treatment for the specific type. Over years several works have been conducted in the area of general automatic segmentation and detection methods of ALL. Most of the methods are based on local image information .Cell segmentation using active contour models is presented in [3].

In [4] proposed a blood image segmentation algorithm based on automatic thresholding and binary filtering .in [5] proposed segmentation technique based on watershed transform to extract the nucleus distribution information is used to extract cytoplasm from the background including
RBC. While effective for nucleus segmentation this method fails when the cytoplasm is not round. Color images allow more reliable image segmentation and provide a better description of a scene than grayscale images [2] many approaches produced several techniques that using transformation of original RGB images into different color space as in [7] proposed algorithm that converts the RGB color space to HSV color space by working on $\mathrm{H}$ channel after extracting it from HSV color space. In [8] it uses Lab color space for segmentation process.

In [9] proposed algorithm Based on HSI color space, enhancement technique .In this paper we proposed a segmentation algorithm on digital microscope images for acute lymphoblastic leukemia based on C-Y color space.

\section{METHODOLOGY}

The main goal of this work is to segment the microscopic image of ALL by converting the RGB color space to C-Y color space after extracting the $\mathrm{Y}$ channel from the C-Y and apply median filter on it .we present a comparison of our algorithm's accuracy and the accuracy of RGB segmentation algorithm.

A. The Dataset-The images of the database have been captured with an optical laboratory microscope coupled with a Canon Power Shot G5 camera. All images are in JPG format with 24 bit color depth, resolution $2592 \times$ 1944. The images are taken with different magnifications of the microscope ranging from 300 to 500. The ALL-IDB database has two versions [10]; ALL-IDB2 version of the database is used.
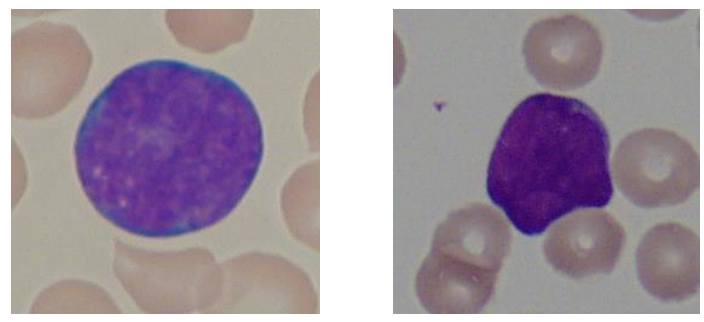

Fig. 1. Example of ALL Dataset

\section{B. C-Y Color space}

Through reviewing several techniques that used transformation of RGB color space to different other color 
space, it has been noted that some color space has complex equations of transforming like HSI. So we used the C-Y color space. The C-Y color model has three color components B-Y, $\mathrm{R}-\mathrm{Y}, \mathrm{G}-\mathrm{Y}$, and one luminance component Y [11]. Only two of three color components are needed to define a color.

The conversion of the RGB color space to $\mathrm{C}-\mathrm{Y}$ color space can be computed using the following $3 \times 3$ transformation matrix as in (1):

$$
\left[\begin{array}{c}
Y \\
R-Y \\
B-y
\end{array}\right]=\left[\begin{array}{ccc}
0.299 & 0.587 & 0.114 \\
0.701 & -0.587 & -0.114 \\
-0.299 & -0.587 & 0.886
\end{array}\right]\left[\begin{array}{l}
R \\
G \\
B
\end{array}\right]
$$

Here, both R-Y and B-Y represent the chromaticity of a color in $\mathrm{C}-\mathrm{Y}$ color space.

$$
\begin{gathered}
S=\sqrt{(R-Y)^{2}+(B-Y)^{2}} \\
\theta=\left\{\begin{array}{cc}
\tan ^{-1}\left(\begin{array}{l}
R-Y \\
B-Y
\end{array}\right) & \text { for } S \neq 0 \\
\text { undefined } & \text { for } S=0
\end{array}\right.
\end{gathered}
$$

In C-Y color model, saturation (S) as in (2) and hue as in (3), $\theta$ can also be derived from the R-Y and B-Y components as above equations.

In order to convert the $\mathrm{C}-\mathrm{Y}$ color space to RGB color space we use the following transformation matrix in (4):

$$
\left[\begin{array}{l}
R \\
G \\
B
\end{array}\right]=\left[\begin{array}{ccc}
0.1 & 0.1 & 0.0 \\
0.1 & -0.509 & -0.194 \\
0.1 & 0.0 & 0.1
\end{array}\right]\left[\begin{array}{c}
\mathrm{Y} \\
\mathrm{R}-\mathrm{Y} \\
\mathrm{B}-\mathrm{Y}
\end{array}\right]
$$

\section{Proposed approach}

The essential goal of acute leukemia blood cell segmentation is to extract structural component such as blast from its complicated background by using C-Y color model. The proposed algorithm for ALL image segmentation is given below.

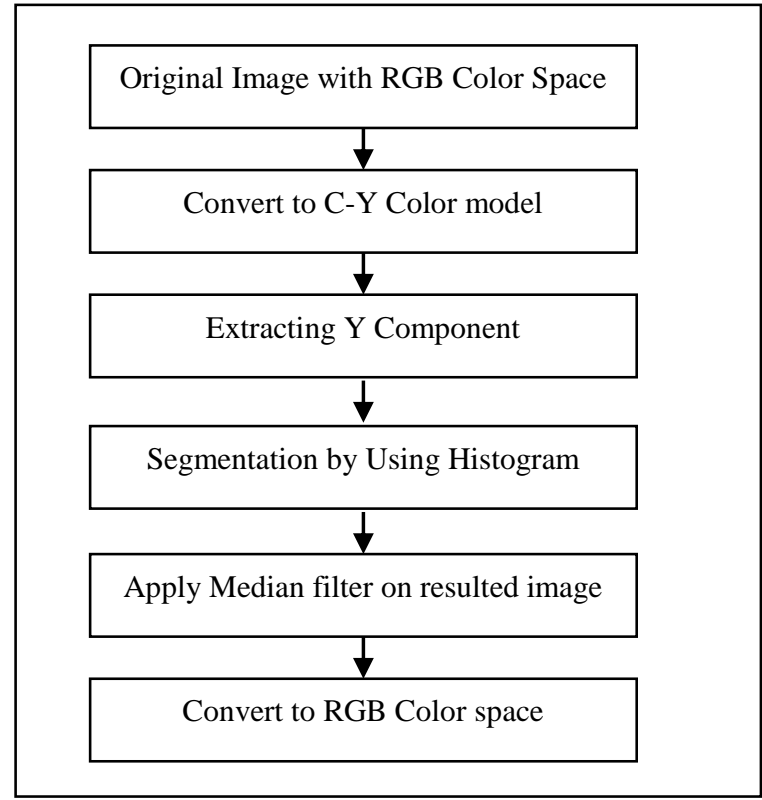

Fig. 2. Proposed system for ALL segmentation
The technique for segmentation ALL by C-Y color model composed of the following steps which are summarized below.

Step 1: transforming the original image with RGB color space to $\mathrm{C}-\mathrm{Y}$ color space using the transformation matrix.

Step 2: Extracting the luminance Y component from C-Y color space.

Step 3: Select the threshold value using Y component from C-Y color space from the histogram.

Step 4: Applying the median filter $\mathrm{N} \times \mathrm{N}(\mathrm{N}=5)$ to the resulted image.

Step 5: Convert the resulted image to RGB \& display the result.

\section{EXPERIMENTAL RESULTS AND DISCUSSION}

In this presented work we introduced an approach of segmentation the acute leukemia blood cell (ALL) based on $\mathrm{C}-\mathrm{Y}$ color space. The result obtained after applying the proposed algorithm as shown below.

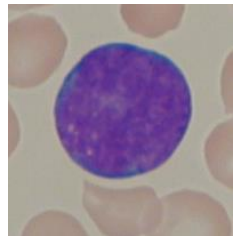

(a)

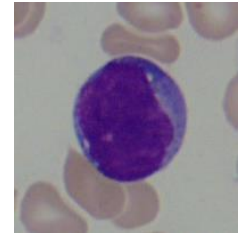

(b)

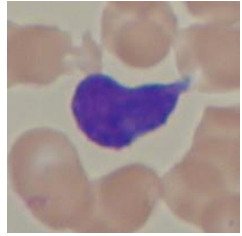

(c)
Fig. 3. are three different examples of original RGB images

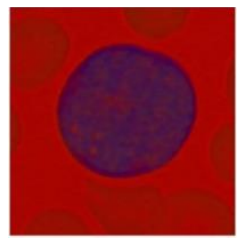

(d)

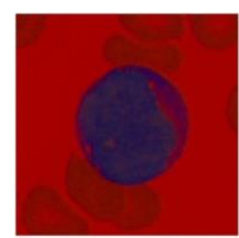

(e)

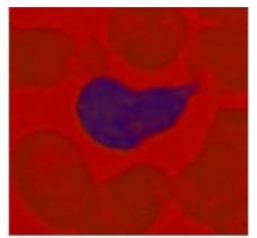

(f)
Fig. 4. (d,e,f) are three Equivalent C-Y Images for a,b,c

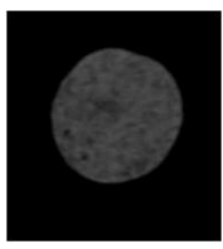

(g)

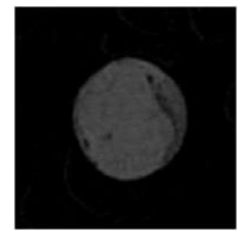

(h)

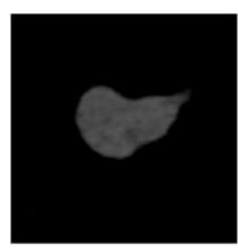

(i)
Fig. 5. $\quad(g, h, i)$ are three B-Y components of C-Y for $d, e, f$

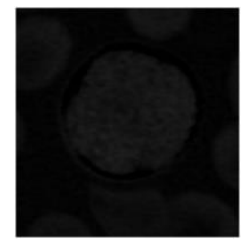

(j)

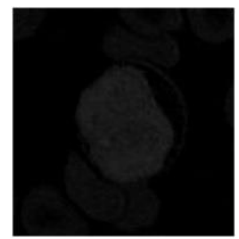

(k)

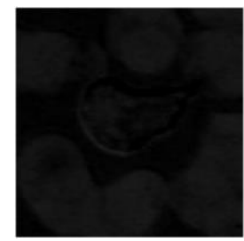

(1)
Fig. 6. (j,k, $)$ are three R-Y components of C-Y for d ,e , f 


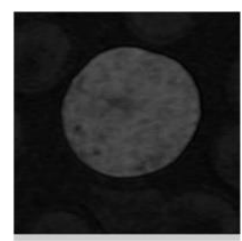

(m)

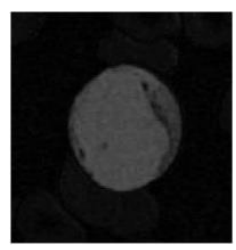

(n)

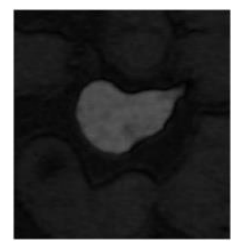

(o)
Fig. 7. (m,n,o ) are three $\mathrm{S}$ components of $\mathrm{C}-\mathrm{Y}$ for $\mathrm{d}, \mathrm{e}, \mathrm{f}$

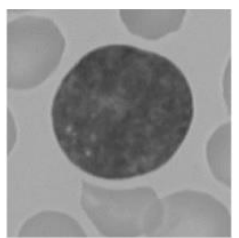

(p)

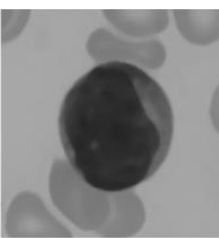

(q)

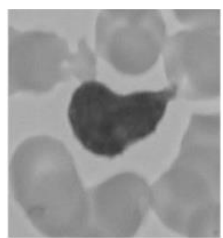

(r)
Fig. 8. (p,q,r ) are three $\mathrm{Y}$ components of $\mathrm{C}-\mathrm{Y}$ for $\mathrm{d}, \mathrm{e}, \mathrm{f}$

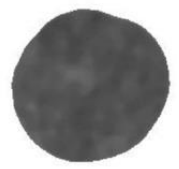

(s)

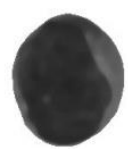

(t)

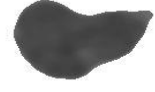

(u)
Fig. 9. (s,t,u) are three segmented images of $\mathrm{Y}$ component $(\mathrm{p}, \mathrm{q}, \mathrm{r})$

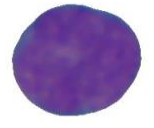

(v)

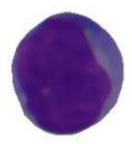

(w)

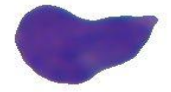

$(\mathrm{x})$
Fig. 10. (v,w,x ) are the converted of $(\mathrm{s}, \mathrm{t}, \mathrm{u})$ images into RGB

The proposed methodology is tested using 100 images of ALL cells and it has successfully isolated the blast part of the cell from the other components of the image this segmentation based on the luminance (Y) component of C-Y color space. To validate the proposed method statistically, the global quantitative method is used. A comparison on area pixels between the accuracy of $\mathrm{C}-\mathrm{Y}$ algorithm and RGB algorithm according to the expert result was done to measure the accuracy of the method in quantitative manner.

This next table shows a performance comparison of acute leukemia image segmentation accuracy using $\mathrm{C}-\mathrm{Y}$ and RGB color spaces. For image segmentation using RGB and $\mathrm{C}-\mathrm{Y}$ color space observation it is found that the method based on RGB color space has not performed well .Means it should not gives accurate result, Besides that ,the shape of the blast after the segmentation process is not quite similar to the expert blasts. While the method based on $\mathrm{C}$-Y color space using $\mathrm{Y}$ component can provide a better accuracy segmentation and shape to the expert blasts.

TABLE I. THE RESUlTS OF SEGMENTATION'S ACCURACY BY C-Y COLOR SPACE COMPARED WITH THE SEGMENTATION'S ACCURACY BY RGB COLOR SPACE

\begin{tabular}{|c|c|}
\hline Type of cell & Segmentation accuracy \\
\hline C-Y & $98.38 \%$ \\
\hline RGB & $95.93 \%$ \\
\hline
\end{tabular}

\section{CONCLUSION}

In this paper we proposed a technique of segmentation of ALL microscope images by using C-Y color space. This segmentation is based on the luminance (Y) component of $\mathrm{C}$ $\mathrm{Y}$ color space. This approach also showed that applying the $\mathrm{C}$ $\mathrm{Y}$ color space is simpler than other color space due to their complex equations and show good result in segmentation of ALL microscopic color images. The segmentation accuracy for the tested images is $98.38 \%$ compared to the accuracy of segmentation of RGB color space $95.93 \%$. The results show that our segmentation technique is robust. In the future, the result of this work can be used as the basis for extracting the other features from the acute lymphoblastic leukemia blood samples.

\section{REFERENCES}

[1] The Cancer Institute of New Jersey Patient Education Committee, Leukemia and Lymphoma awareness month , New Jersey, USA, 2008.

[2] Aimi Salihah , A.N, M.Y.Mashor, Nor Hazlyna Harun “ Colour Image Enhancement Techniques for Acute Leukemia Blood Cell Morphological Features “, IEEE pp.3677-3682, 2010.

[3] G. Ongun. U. Halici. K. Leblebicioglu . V. Atalay. M. Beksac , and S. Beksac." An automated Differential Blood Count System" .In Int.Conf. off IEEE Engineering in Medicine and Biology society, Volume 3, pages

[4] Angulo J, Flandrin G. Microscopic image analysis using mathematical morphology: application to haematological cytology, 1:304-312, 2003.

[5] Dorini LB, Minetto R, Leite NJ. White blood cell segmentation using morphological operators and scale-space analysis. Proc Brazilian Symp Comput Graph Image Proc, 294-304, 2007.

[6] M. Veluchamy, K. Perumal and T. Ponuchamy, "Feature Extraction and Classification of Blood Cells Using Artificial Neural Network," American Journal of Applied Science, vol. 9, pp. 615-619, 2012.

[7] N.Sinha and A. G. Ramakrishman. Automation of Differential Blood Count. In Proceeding Conference on Convergent technologies for Asia pacific Region .2:547-551.2003.

[8] S. Mohapatra and D. Patra, "Automated Cell Nucleus Segmentation and Acute Leukemia Detection in Blood Microscopic Images," in International Conference on Systems in Medecine and Biology, India, 2010 .

[9] N. H. A. Halim, M. Y. Mashor, A. S. Abdul Nasir, N. R. Mokhtar, and H. Rosline. 2011. Nucleus segmentation technique for acute leukemia, in Proceedings of the IEEE 7th International Colloquium on Signal Processing and Its Applications (CSPA '11), pp. 192-197, March 2011.

[10] Donida Labati, R., Piuri, V., Scotti, F. ALL-IDB:the Acute Lymphoblastic Leukemia Image DataBase for image processing, 2011.

[11] E. R. Dougherty, Electronic Imaging Technology, SPIE-The International Society for Optical Engineering, 1999. 2583- 2586.2001. 